\title{
TP53 Gene Inactivation
}

National Cancer Institute

\section{Source}

National Cancer Institute. TP53 Gene Inactivation. NCI Thesaurus. Code C36329.

A mutation in the TP53 gene that either inhibits the expression of or results in the translation of an inactive cellular tumor antigen p53 protein. 